\title{
Circuit
}

Musiques contemporaines

\section{Saison 1992-1993 : l'année de la nostalgie}

\section{Dominique Olivier}

Volume 5, numéro 1, 1994

Gilles Tremblay : réflexions

URI : https://id.erudit.org/iderudit/902099ar

DOI : https://doi.org/10.7202/902099ar

Aller au sommaire du numéro

Éditeur(s)

Les Presses de l'Université de Montréal

ISSN

1183-1693 (imprimé)

1488-9692 (numérique)

Découvrir la revue

Citer ce compte rendu

Olivier, D. (1994). Compte rendu de [Saison 1992-1993 : l'année de la nostalgie].

Circuit, 5(1), 79-100. https://doi.org/10.7202/902099ar
Résumé de l'article

Proposant comme à l'accoutumé un panorama critique de la saison montréalaise 1992-1993, l'auteure insiste sur ses caractéristiques marquantes : peu de jeunes compositeurs ont été joués; la nostalgie du déjà connu semble refléter l'angoisse actuelle de la société; pour des raisons idéologiques diverses, nos créateurs se sont beaucoup appuyés sur des textes littéraires et religieux. Une œuvre, cependant, surnage de ce marasme : L'adorable verrotière, de Serge Provost. d'utilisation que vous pouvez consulter en ligne.

https://apropos.erudit.org/fr/usagers/politique-dutilisation/ 


\section{Saison 1992-1993: l'année de la nostalgie Dominique Olivier}

Si l'on tient à tout prix à donner un visage à cette saison musicale « contemporaine» montréalaise de 1992-1993, c'est celui de la nostalgie qui s'impose avec le plus de force à notre imagination. Non pas une nostalgie exprimée par l'attitude postmoderne caractéristique de ces dernières années, mais une véritable tristesse «causée par l'éloignement de ce que l'on a connu, par le sentiment d'un passé révolu, par un désir insatisfait ", comme la définit le dictionnaire (Larousse, 1977). On ne récupère plus pour faire du neuf: on cherche à se berner en recréant tel quel ce qui constituait le fruit d'une véritable activité créatrice, motivée sans doute par la sensation d'aller vers quelque chose. Si l'on prend pour acquis - on ne peut guère le nier, à moins de nager dans une bienheureuse inconscience - que la musique, comme les autres arts, évolue en fonction de la société dans laquelle elle est réalisée, il serait bien curieux qu'elle ne vive pas une crise profonde ("). Dans ce cas, il serait également bien étrange que le monde de la création ne se tourne pas derechef vers ce qui fut, à défaut de pouvoir fabriquer ce qui sera. Tout autant que par le passé, le milieu de la création - tant au Québec que partout ailleurs - joue le rôle d'un catalyseur et d'un reflet de ce qui l'environne. Si l'angoisse devient dominante, elle ne peut que transparaître dans l'ensemble de cet organisme vivant qu'est le monde de la création musicale. Et devant cette angoisse, provoquée non plus par un désengagement dangereux mais réversible, mais plutôt par un renoncement de plus en plus explicite à tout ce qui n'est pas éminemment rentable, on «nostalgise ». Hétu, Pépin, Tremblay, Xenakis et Kagel ont occupé le devant de la scène musicale cette dernière saison, comme si les créateurs, tout autant que les organisateurs de concerts, voulaient nous faire sentir le péril, le danger d'une mort potentielle du milieu, qui pourrait, de forum, devenir musée. Peutêtre est-ce là un pessimisme de mauvais aloi, un alarmisme inutile, facilement récusables par la toute puissante loi du balancier. On peut néanmoins constater que peu de jeunes créateurs
(1) En témoignent d'ailleurs, entre autres, un débat publié dans le monde de la musique de février 93, qui réunissait les opinions de trois critiques musicaux français, ainsi qu'un dossier de la revue Diapason de novembre 1993, intitulé - La musique contemporaine est morte, vive la nouvelle musique ? », signe que la question ne préoccupe pas seulement les spécialistes, mais aussi le grand public mélomane. 


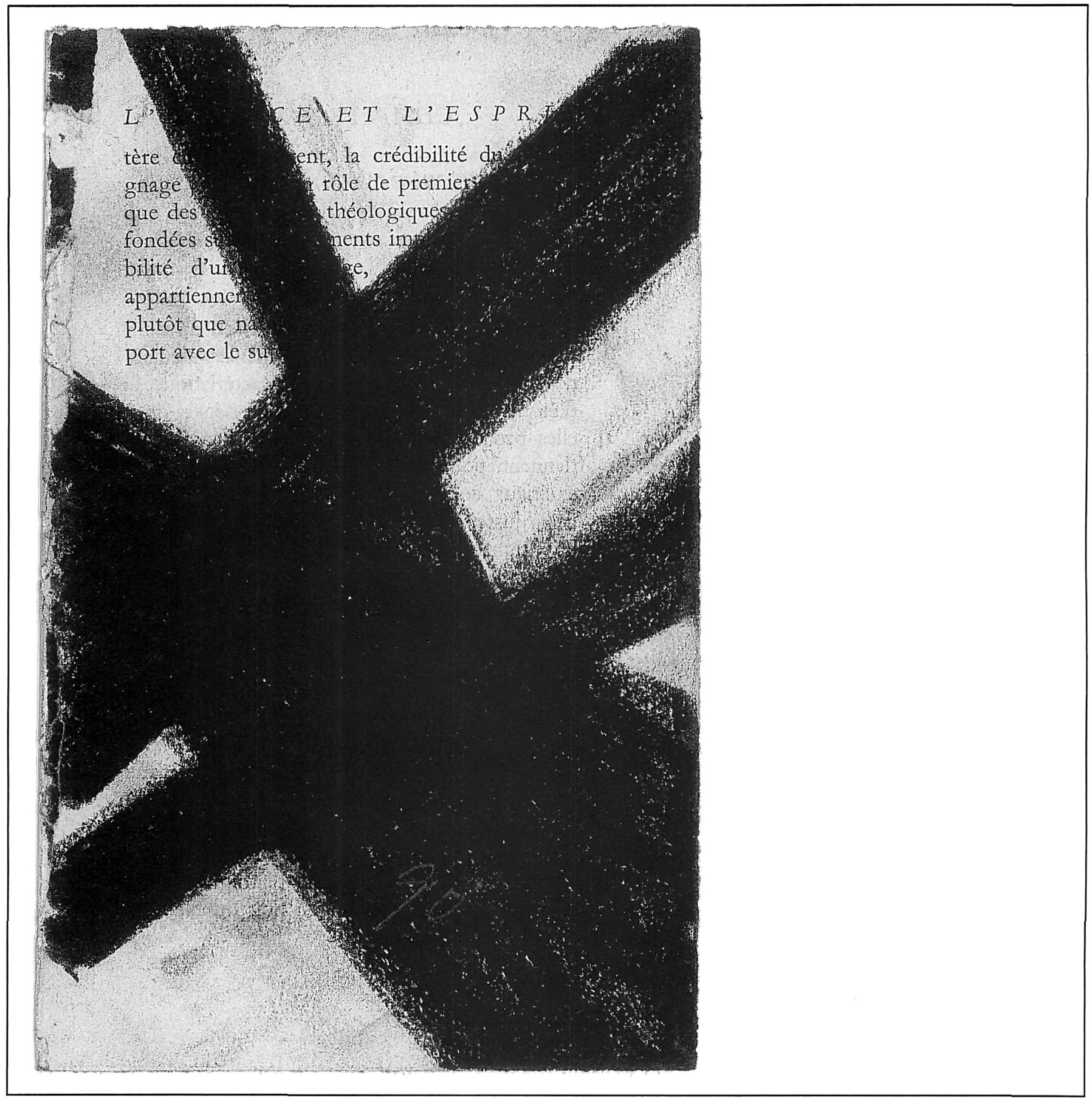


ont été joués durant cette grisonnante saison, et que les conservatismes musicaux et idéologiques, véhiculés avec ostentation par certains membres de «la vieille garde », y avaient l'air de véritables capitulations. Après l'abandon des valeurs traditionnelles, la résurgence de celles-ci devraitelle nous sembler salvatrice ? Elle devrait plutôt nous inquiéter.

Un autre fait remarquable, dans cette saison musicale contemporaine, est le grand usage qu'ont fait les compositeurs de textes littéraires, religieux ou destinés à l'opéra. Déjà, la création de trois «opéras " (L'adorable verrotière de Serge Provost, Le Prix de Jacques Hétu et II suffit d'un peu d'air de Claude Ballif) dans la même saison est un fait marquant, alors que la création de deux autres œuvres d'envergure (Messe sur le monde de Clermont Pépin et Avec de Gilles Tremblay), avec chœur, orchestre et solistes chanteurs ajoutaient encore à ce fait étonnant. $D^{\prime}$ autres créateurs, jeunes et moins jeunes, ont utilisé eux aussi le support du texte, soit Sylvaine Martin avec Adolescence, Isabelle Panneton avec la Cantate de la fin du jour, Jacques Desjardins avec Décimations ou Denis Gougeon, dans sa Complainte de la passion. Avec l'événement Kagel, on donnait également une place considérable au théâtre musical, produisant ainsi une saison où «l'impureté » de l'inspiration semblait caractéristique. II s'agit peutêtre d'une nouvelle avenue, d'un complément nécessaire au travail du compositeur. Mais encore plus insidieusement qu'un langage musical, le choix d'un texte peut orienter l'écoute et diriger l'auditeur dans des voies qui sont, ou ne sont pas, souhaitables.

\section{La saison du NEM}

II est difficile ici de parler d'une saison au sens habituel du terme, puisque le NEM participait, en 92-93, à plusieurs événements qui n'étaient pas tous regroupés sous la même bannière. Les deux opéras auquel l'ensemble s'est associé, soit L'adorable verrotière et II suffit d'un peu d'air, étaient organisés respectivement par l'Académie des lettres et des sciences humaines de la Société Royale du Canada et par la Compagnie lyrique de création « Chants libres», en coproduction avec le NEM. Outre l'événement Kagel 92, seulement deux concerts restaient dans l'esprit de la saison traditionnelle: le Concert des solistes et le Grand concert classique. Plus que jamais, le NEM s'est affirmé non pas comme une société de concert, mais comme un atelier de création et d'interprétation de la musique $d u X X^{e}$ siècle, un lieu d'expéri- 
mentation et de respect du passé tout à la fois, apportant l'oxygène nécessaire à la survie d'un immense corpus culturel, celui de la musique dite contemporaine, née dans un siècle à la veille de l'achèvement.

\section{L'adorable verrotière}

Dans le cadre du colloque sur la musique ef les arts de la scène aujourd'hui, organisé par la Société Royale du Canada ${ }^{(2)}$, nous avons pu entendre, en tout début de saison, soit le 3 septembre 1992, ce qui allait rester le point culminant de toute l'année musicale, L'adorable verrotière, du compositeur montréalais Serge Provost. Plus encore : non seulement cet « opéra , au sens litigieux du terme, est-il la création la plus marquante, la plus émouvante de la saison 92-93, mais nous croyons pouvoir dire qu'il s'agit d'une des œuvres les plus importantes que le Québec ait produite, au même titre que ...chant d'amours de Serge Garant, ou Kopernikus de Claude Vivier. Sur des extraits d'un texte du poète montréalais Claude Gauvreau, Le Vampire et la nymphomane, écrit en 1949 et destiné à un opéra que devait écrire le regretté Pierre Mercure-, ou plutôt « avec » ce texte, Provost a su créer un moment d'émotion intense qui s'apparente à une plongée au cœur du sens même de la musique. Tant par la structure, d'une rigueur sans compromis, que par la symbiose exceptionnelle qu'il réussit à obtenir entre les mots et les sons, le compositeur nous donne accès à la « musique » de Gauvreau, au sens caché derrière les mots, ce sens qui ne veut rien dire (Provost 1992: 21)... La déconstruction opérée par Gauvreau ramène le langage à son état premier, * où les mots-signes trouveraient leur seule motivation possible qui résiderait dans leurs valeurs ou dans leurs connotations émotionnelles » (Fisette 1992: 12). Le résultat obtenu par Gauvreau en fait un musicien dont l'œuvre peut encore gagner à être mise en musique, si cette dernière s'apparente réellement à la sensibilité du poète. Cette parenté de sensibilité entre le compositeur Serge Provost et le poète Claude Gauvreau s'exprime par une volonté d'échapper à la figuration, à l'anecdote, au "premier niveau ", ainsi que par une intransigeance face à l'intelligibilité du message. Au-delà de toutes ces considérations, cependant, ce qui reste de L'adorable verrotière, c'est la sensation d'avoir vécu un moment privilégié de conscience, un de ces moments inexplicables qui nous laisse ébranlé, sans voix, sans mots pour dire l'émotion. Si le texte de Gauvreau, très dur, violent même, semblait nous labourer l'intellect à coups de griffes acérées, la musique admirable donnait à cette « agression » une dimension qui l'éloignait complètement de la trivialité présente dans nos propres références à ces mots. En même temps que symbiose, il y avait
(2) Les contributions de Jean Fisette, de Serge Provost et de Pauline Vaillancourt à ce colloque ont été publiées dans le volume III, no 2 de CIRCUIT. 
dichotomie, effet doublement déchirant. Pauline Vaillancourt, tragédienne lyrique qui fut, à cette occasion, une interprète d'une intensité encore plus troublante qu'à l'accoutumée, mérite certainement une grande partie des éloges accordés à l'œuvre. Le Nouvel Ensemble Moderne fut exemplaire, tout à fait à la hauteur de sa tâche. Si l'œuvre de Gauvreau - tout comme celle de Provost - est une partition, un ensemble d'instructions permettant aux interprètes de provoquer la venue d'un moment de beauté (selon Fisette), cette partition fut plus que respectée, elle fut ce soir-là transcendée. L'absence de mise en scène, malgré l'appellation d'opéra, était tout à fait approprié à l'esprit de l'œuvre.

\section{Kagel, biennale 92 du NEM}

Pour un groupe de musiciens, pouvoir consacrer une dizaine de jours consécutifs à honorer un compositeur vivant, dans le contexte actuel, est déjà une provesse. Si l'événement est par ailleurs une réussite, on peut presque crier au miracle. La Biennale 92 du NEM (qui s'est tenue du 4 au 14 novembre) a été ce miracle, malgré le fait qu'il s'agissait de la première expérience du genre pour l'ensemble. Dix-neuf éléments constituaient la très riche programmation de cette biennale, qui mettait en lumière les nombreuses facettes de ce créateur pluriel, Mauricio Kagel. Ce choix, s'il était dicté d'abord par les contingences matérielles, s'explique aussi par le rôle fondamental joué par Kagel au sein de l'avant-garde musicale, pendant ces années que l'on pourrait qualifier de fastes. Si aujourd'hui on lui rend hommage, ce n'est pas bien sûr à titre posthume, mais non sans une certaine trace de nostalgie pour ce qui fut une époque de constante remise en cause, de reconstruction perpétuelle. Mais Kagel, puisqu'il n'a pas suivi la voie du sérialisme et du structuralisme, issus du pseudo-néant de la tabula rasa, est peutêtre un postmoderne avant la lettre, un de ces créateurs qui n'ont pas renié leurs racines culturelles, mais qui sont au contraire allé les contempler avec une lucidité exceptionnelle. C'est là sans doute que réside son génie, beaucoup plus que dans l'accomplissement de ses œuvres musicales et théâtrales, qui, si elles sont efficaces, ne sont pas marquantes individuellement. L'intérêt de cette biennale à laquelle le compositeur a participé activement, était de nous permettre de découvrir ce que représentait au juste Kagel, pour les jeunes comme pour les stigmatisés de l'avant-garde.

Théâtre musical, concerts, films, rencontres et répétitions publiques constituaient le corps de cette biennale, dont le centre a été pour nous la présen- 
tation de La Trahison orale. Au cœur de l'œuvre de Kagel, le théâtre musical occupe une place privilégiée, puisqu'il intègre la «composition avec un matériau non-résonnant», un travail cher au compositeur, qui établit toutefois une distinction entre théâtre instrumental et théâtre musical. Dans La Trahison orale, théâtre musical, l'œuvre n'existe pas sans le support de la musique. Omniprésente et personnage à part entière, elle réussit, au même titre que le texte, conçu par Kagel, à créer un effet d'envoûtement très particulier. Car Kagel y utilise au maximum un outil d'une efficacité éprouvée, la référence culturelle. Le texte, tout d'abord, est construit sur la base de légendes, de contes, d'anecdotes se rapportant au diable, mais adapté selon le lieu des représentations. On pouvait ainsi goûter à des références ayant un fort relent de vieille campagne québécoise. La musique, elle, reste toujours fidèle à la partition, mais utilise également un fond commun de culture sonore habilement exploité par Kagel, pour créer l'état émotif approprié. Insidieusement, les sons y travaillent à nous orienter, à nous mettre dans l'angoisse ou l'hilarité, selon le désir du compositeur. Un peu plus avant, le spectateur, au départ impuissant et victime de son dictionnaire intérieur, peut prendre du recul, et constater l'intelligence de celui qui, par sa grande habileté à percevoir ce qui fait les lieux communs d'une culture, nous met en face de nous même, avec humour. Puisqu'on ne peut échapper à certains déterminismes, le mieux est, certes, d'en prendre conscience, nous démontre Mauricio Kagel dans ses meilleures œuvres. Le côté bouffon du personnage, son manque de sérieux apparent, cachent un sens aigu de l'observation, et une capacité de synthétiser l'essence d'un morceau de culture humaine.

Les autres concerts de la biennale présentaient un moins grand intérêt, mais avaient le mérite de nous montrer différents aspects du musicien Kagel. Le concert d'ouverture, dirigé par Lorraine Vaillancourt, offrait une des œuvres instrumentales majeures du compositeur, 1898, déjà interprété par le NEM lors du premier Grand concert classique, ainsi que Zwei Akte, pour harpe et saxophone, une pièce qui, encore une fois, est constituée de clichés véhiculés par les répertoires respectifs de ces deux instruments, censés incarner la masculinité et la féminité. Un peu puéril, peutêtre... Avec Variétés, interprété par les musiciens de l'Atelier de musique contemporaine de la Faculté de musique de I'Université de Montréal, Kagel lorgne, à l'instar de Fellini, du côté du cirque, univers fascinant où la musique a toujours joué un rôle strictement utilitaire. L'aspect visuel et divertissant y est donc nettement prédominant. Avec Variétés, on s'amuse, et c'est tout. Avec Dressur, interprétée au dernier concert de la biennale du NEM, dirigée par Kagel lui-même, cette sensation persiste. Trois percussionnistes s'y livrent à de curieux agissements n'ayant que très peu à 
voir avec la musique, dans un but plutôt nébuleux, qui rejoint sans doute une volonté de remettre en question le rôle de l'instrumentiste dans le concert traditionnel. Quatre extraits de la Rose des vents, ainsi que Phantasiestück, complétaient ce concert-spectacle qui nous a laissé sur notre faim de musique. Définitivement, le génie de Kagel, si génie il y a, n'est pas musical. II réside dans sa faculté à engendrer un climat de dérision, créant un recul salutaire par rapport à la tradition, une tradition qu'il utilise sans scrupule avec un plaisir de petit garçon découvrant les jeux de blocs...

\section{Il suffit d'un peu d'air}

Parmi toutes les voies que l'art lyrique a explorées au cours des dernières décennies, celle de la trivialité n'est certes pas la plus exaltante. La création en novembre 92 de l'opéra II suffit d'un peu d'air, de Claude Ballif, sur un texte de Renald Tremblay, nous a permis de le constater avec force. "Chants libres», compagnie lyrique de création, a le mérite de chercher de nouvelles avenues pour cet art constamment en déconstruction qu'est l'opéra. Ce que lui propose l'association Ballif-Tremblay n'a rien qui puisse l'aider à gagner la faveur d'un quelconque public, qu'il soit traditionaliste ou féru de musique contemporaine. S'il était malheureusement impossible pour «Chants libres» de refuser une œuvre commandée, l'ennui dégagé par II suffit d'un peu d'air est tel, qu'elle semble avoir été écrite pour démontrer pédagogiquement ce que l'on doit faire pour décourager l'auditeur le plus rempli de bonne volonté. Le texte dégage un fumet de banalité exaspérant, alors que la musique distille un ennui abyssal que les plus grands interprètes n'arriveraient pas à occulter. $\mathrm{Si}$ les protagonistes de cette histoire cherchent à fuir l'angoisse par un usage excessif de la parole, ne serait-il pas plus pertinent de fuir ce genre d'irritant que l'on rencontre malheureusement un peu trop souvent dans la vie quotidienne? Pauline Vaillancourt, Renée Lapointe, David Doane, Michel Ducharme et le NEM n'y pouvaient rien, seule manquait l'inspiration des auteurs...

\section{Concert des solistes et Grand concert classique}

En décembre 92, le Nouvel Ensemble Moderne présentait un de ses événements récurrents, le concert des solistes qui met en vedette ces interprètes voués en général à une plus grande discrétion. C'est l'occasion d'entendre individuellement le jeu de ces musiciens qui, réunis, forment un 
ensemble d'une unité et d'une force exceptionnelles. C'est autour de Denis Gougeon qu'ils se sont cette fois regroupés, tout en restant des solistes à part entière. Six œuvres de Gougeon, formant un cycle appelé Six thèmes solaires, formaient l'essentiel de ce programme où l'on pouvait entendre également du Richard Boucher, du Alice Ping Ye Ho, du Petros Shoujounian et du Rodney Sharman. Un concert agréable et sympathique, somme toute, mais sans grande révélation.

Le Grand concert classique nous revenait en avril après deux ans d'absence, comblant un besoin que le NEM a lui même créé, depuis 1989. Si la nostalgie était au rendez-vous à cet événement Xenakis d'une durée de quatre jours - où l'on ressortait presque avec la larme à l'œil les souvenirs associés au rôle de Xenakis dans l'éveil de la modernité au Québec -, ce bain de modernité proposé par différents organisateurs était d'une qualité enrichissante. Pour clore cet événement, le NEM offrait par son concert classique un hommage à ce créateur, plus musicien que mathématicien. Pour créer un intérêt supplémentaire, trois manières de faire la musique au $X X^{\bullet}$ siècle étaient, dans le programme, mises en parallèle. Celle de Xenakis, bien sûr, mais aussi celle de Serge Garant et de Giacinto Scelsi. Quoi de plus différents que ces trois créateurs : le premier à la recherche d'une nouvelle manière de composer extrêmement complexe, s'apparentant plus aux mathématiques qu'au métier de musicien, mais réussissant toujours à écrire des œuvres accessibles, musicales, comme s'il partait sur une piste indéchiffrable et finissait toujours par suivre son intuition... Le second, émule de Webern, grand défenseur du dodécaphonisme, structuraliste orthodoxe, d'un esprit organisé capable de suivre une voie déjà tracée, mais aussi de la prolonger encore, de l'amener à un degré de perfection ne reniant pas l'expressivité. Le dernier, assez insignifiant, essaye de faire croire par des effets nébuleux à la présence d'une pensée profonde dans sa musique, cachée derrière des sons censés nous appeler à la contemplation... Faire s'entrechoquer ces trois conceptions musicales était un coup de génie, que nous devons à Lorraine Vaillancourt, qui a dirigé ce soir-là ses musiciens avec une tension soutenue et une intensité qui faisait de cet événement un grand moment de musique. 


\section{Événements isolés}

\section{Avec, Wampum symphonique}

A l'occasion du $350^{\circ}$ anniversaire de la fondation de Montréal, RadioCanada produisait, le 12 octobre 92, un événement musical d'envergure comprenant la création d'une œuvre de circonstance dont l'élaboration avait été confiée au compositeur Gilles Tremblay. Tremblay, bien entendu, était tout destiné pour réaliser cette œuvre grandiose qui devait couronner les célébrations montréalaises, et être diffusée en direct dans plus de vingt pays. Avec, Wampum symphonique pour soprano, basse, récitant, chœur mixte et grand orchestre, est une œuvre considérable par les effectifs, et impressionnante par les effets employés. L'Orchestre Symphonique de Montréal, dirigé par Charles Dutoit, le chœur Saint-Laurent préparé par Iwan Edwards, ainsi que les solistes Marie-Danielle Parent, Claude Corbeil et Hubert Gagnon (le récitant), ont donné une interprétation émouvante de ce «wampum», dans une église Saint-Jean-Baptiste remplie d'auditeurs enthousiastes. En repensant à l'événement, on peut toutefois émettre certaines réserves quant au sujet traité. Musicalement, Tremblay s'est contenté d'écrire dans son langage habituel, très coloré et facilement identifiable. La réutilisation de Musique du feu, créée en 1991 par la SMCQ et orchestrée pour s'intégrer à $A v e c$, était une solution de facilité, certes, mais convenable. II s'agissait, comme nous l'avons dit précédemment, d'une œuvre de circonstance, qui n'allait pas laisser d'autres souvenirs que ceux d'un moment de célébration plutôt réussi, se terminant par un tremblement de terre musical suivi d'un long silence. Les textes choisis, quant à eux, montraient un autre visage du créateur. La bonne conscience se porte très haut, ces années-ci, et Avec n'est pas étranger à cette tendance... L'appellation de «Wampum», terme utilisé par les Amérindiens - premiers habitants de ce continent, n'est-il pas vrai ? -, et désignant un objet, ceinture ou collier à motifs symboliques, fabriqué à des fins de réconciliation, donne déjà le ton. Le mot avec fait évidemment allusion à la volonté d'établir le lien avec autrui. Des extraits de textes à résonances religieuses, en contrepoint avec des phrases de wampum, forment la trame de l'œuvre. L'histoire de Montréal, si elle est indissociable de ses fondateurs - dont ce Royer de la Dauversière, mystique et utopiste, nous dit Tremblay-, comporte pourtant une foule d'autres aspects qui auraient pu tout aussi bien être mis en évidence. Pour justifier ce choix, on peut invoquer la forte présence de l'esprit religieux 
dans la société canadienne-française, de ses débuts jusqu'à tout récemment, ou alléguer l'influence considérable du clergé sur le développement de cette société. Raison de plus pour éviter de s'y complaire, pourrait-on également défendre...

\section{Le Prix}

L'opéra Le Prix, de Jacques Hétu sur un texte d'Yves Beauchemin, créé au printemps 1993, a été faussement accueilli comme le premier opéra québécois. Il pourrait, par contre, être considéré à bon droit comme le premier opéra «traditionnel " de fabrication entièrement québécoise. Car dans sa facture, Le Prix reste beaucoup plus près du théâtre lyrique conventionnel que d'un Ne blâmez jamais les Bédouins, par exemple. L'aspect conservateur de l'œuvre devait, en tant que critère absolu, permettre à un public nombreux de venir assister à une véritable création. Orchestre, chanteurs, costumes, décors, tout y était, avec la petite note triviale qui devait nous permettre de nous identifier à l'œuvre, nous, citoyens nord-américains de la fin du XXe siècle, tout imprégnés de culture télévisuelle, pour ne pas dire de culture "téléromantique ». Tout aurait pu en rester là, si les auteurs n'avaient pas fait preuve de finesse et d'intelligence. Hétu et Beauchemin ont su, dans le cadre de cet opéra en apparence banal, relier de manière presque indécente à notre quotidien, user de délicatesse et de recul. Les couleurs orchestrales tant vantées, la justesse de l'expression dramatique, ainsi que le choix de Jacques Hétu d'écrire pour un interprète en particulier, en l'occurrence la basse Joseph Rouleau, rendait la chose plus que simplement valable. Yves Beauchemin, quant à lui, a fait preuve d'assez d'humour et d'ironie discrètement mordante pour nous rendre son texte sympathique. Le Prix est somme toute une œuvre sans prétention, et contemporaine - plus par sa date de création que par sa facture -, sans être dérangeante.

\section{Deux créations de Denis Gougeon}

Deux œuvres du très prolifique compositeur montréalais Denis Gougeon étaient créées à quelques jours de distance, au printemps de cette saison 1992-93. Tout d'abord, le 27 avril, Fragile fixe fugace était donné par l'Orchestre Symphonique de Montréal, alors que la Complainte de la passion était interprétée pour la première fois le 13 mai, par le Quatuor Morency et le soprano Marie-Danielle Parent. Le langage chatoyant, expressif et essentiellement mélodique de Denis Gougeon produit, côte à côte, des œuvres 
poignantes et des objets musicaux sans grand intérêt, comme ce Fragile fixe fugace qui fut un des moments faibles de la saison. II faudrait sans doute réentendre cette œuvre orchestrale dans de meilleures conditions, puisqu'il arrive fréquemment que l'OSM nous serve, en musique contemporaine, l'équivalent d'une première lecture des pièces. La Complainte de la passion, de son côté, offrait un tout autre spectacle, car les interprètes y étaient non seulement bien préparés, mais également très impliqués. Marie-Danielle Parent, avec sa grande générosité musicale et dramatique, a su véritablement faire revivre cette douleur de la mort du Christ, mise en musique par Gougeon, avec des accents de souffrance maternelle tout à fait poignants. Le Quatuor Morency, familier avec la musique du XX $X^{e}$ siècle, jouait avec beaucoup d'intensité ce drame de la passion qui, notons-le, nous met pour la seconde fois devant un sujet religieux.

\section{La Messe sur le monde}

Les évocations religieuses ef la complaisance passéiste caractéristiques de plusieurs créations entendues au cours de l'année ne nous avaient pas encore complètement accablées, jusqu'à la date du 28 avril 1993, journée de la création, à Québec, de La Messe sur le monde (Symphonie no. 4), de Clermont Pépin. Ce jour-là, la saison prenait une teinte désespérante, d'autant plus que l'on avait donné à cet événement d'importance une visibilité rarement accordée à la musique récente. Jusqu'à la nausée, Clermont Pépin nous a servi ce soir-là sa vision réactionnaire du monde, un monde sur lequel se déroulait une messe à la mémoire de la musique... Cette œuvre malsaine donnait à voir une conception tout à fait écœurante d'un univers qui, au seuil de sa décadence, s'accroche désespérément à tout ce qui peut le faire chuter encore plus vite. L'obscurantisme, à ce que nous en sachions, n'était plus considéré ces derniers temps comme une solution à la misère humaine ef à l'appauvrissement culturel généralisé. Et depuis belle lurette, Teilhard de Chardin n'est plus perçu comme une sommité dans le domaine scientifique. Les textes employés, extraits entre autres de Teilhard, allaient aussi loin qu'il ne le faut pas dans la voie de l'abnégation. Qu'on en juge par cet extrait: «Et maintenant, Jésus, que voilé sous les puissances du monde, vous êtes devenu tout pour moi, enfermez-moi au plus profond de votre Cœur. Et, quand vous m'y tiendrez, brûlez-moi, purifiez-moi, enflammez-moi, sublimez-moi, jusqu'à la satisfaction parfaite de vos goûts, jusqu'à la plus complète annihilation de moimême. " Les sombres gargouillis de Joseph Rouleau, sacré grand prêtre pour l'occasion, donnaient des frissons dans le dos, et le langage orchestral 
sous-tendant cette profession de foi annihilante n'avait rien de plus lumineux que les textes employés. Honegger, Stravinsky, Ravel et Messiaen s'y retrouvaient comme chez eux, baignés dans un langage qui tenait plus du remâchage que du postmodernisme. Les chœurs et l'Orchestre symphonique de Québec, dirigés par Pascal Verrot, étaient par ailleurs bien préparés, ce qui n'augmentait en rien le plaisir qu'on avait à écouter cette soit-disant messe sur le monde. Tout, dans cette œuvre homogène, contribuait à l'effet de noirceur, et exaltait la vision pessimiste d'un monde qui ne peut exister sans le secours divin. Peutêtre, finalement, est-ce la peur qui s'exprime ainsi?

\section{Trois jours avant}

La société «Codes d'accès», qui remplace désormais la Société des concerts alternatifs du Québec (SCAQ), offrait le 5 mars 1993 un concert dont la conception était étroitement reliée avec l'imminence de la fête des femmes: seulement des œuvres de femmes, interprétées surtout par des femmes, et dirigées, dans plusieurs cas, par une femme, la chef d'orchestre Véronique Lacroix. Marie Pelletier, Maya Badian, Ka-Ming Lo, Pascale Trudel, Linda Bouchard et Sylvaine Martin étaient au programme de cette soirée très particulière. Le fait qu'une œuvre soit écrite par une femme ne garantit pas, pas plus que l'inverse, la grande qualité de la production musicale. Le meilleur y côtoyait donc le moins bon, tout comme dans n'importe quel concert du genre. Nous avons retenu surtout le très beau Transi-Blanc, de Linda Bouchard, un Moine à grosse tête et la bête, très original, de la compositrice Ka-Ming Lo, et Adolescence, de Sylvaine Martin, une commande de RadioCanada pour l'occasion. Cette dernière œuvre, toute en demi-teintes et très émouvante, est d'une facture sobre et presque classique, malgré son langage très actuel. Comme beaucoup d'autres créations de la saison, Adolescence s'inspire d'un texte, écrit par la compositrice elle-même, qui justifie ainsi son choix: «Pour ma part une œuvre «à thème » permet de s'investir autrement dans la fabrication de la pièce. On a une orientation extra-musicale qui définit une liberté et une contrainte nouvelles. Ici le texte qui sert de support à la trame musicale raconte un émoi, un instant fugitif que seuls les adolescents savent faire surgir ». La sensibilité toute en délicatesse de Sylvaine Martin a fait de cette œuvre, poème et musique, un très beau moment d'émotion qui, si c'est possible, ressemble peutêtre à ce qu'une femme peut livrer de distinctif par la création musicale. 


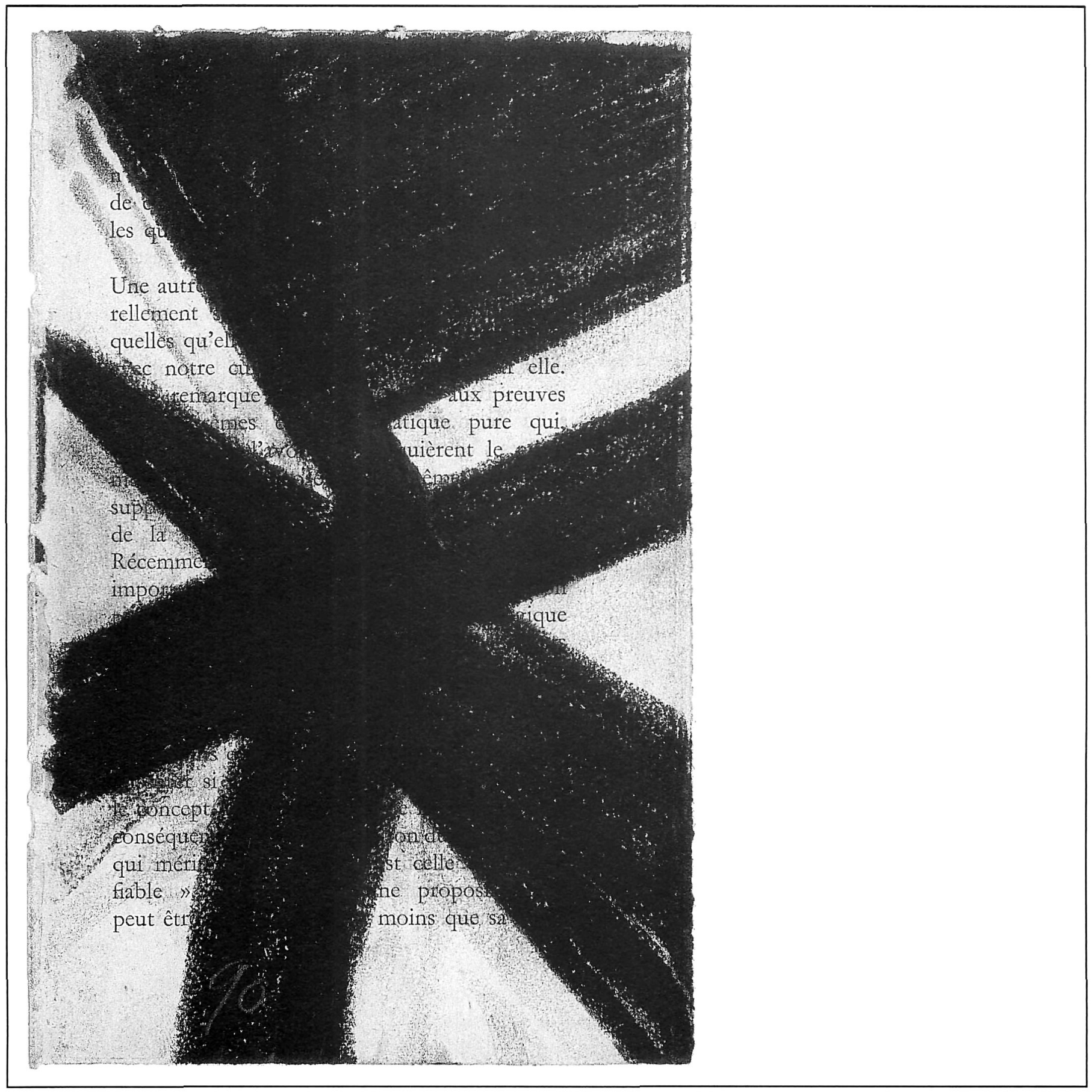




\section{Trois voix chanteras!}

Jean-Sébastien Bach, Jacques Desjardins et Isabelle Panneton étaient, pour I'Ensemble Contemporain de Montréal, le soir du 8 juin 1993, les trois voix qui chantaient, afin de créer le lien entre la grande tradition musicale et le langage des compositeurs d'aujourd'hui. Comme toujours dans les programmes de l'ECM, une œuvre «classique " servait de point de départ à un concert consacré à la création musicale québécoise. la Cantate BWV 4, *Christ lag in Todesbanden », ainsi que deux extraits de la Missa Secunda de Hans Leo Hassler, offraient ce point de départ, vocal il va sans dire. la seconde partie était consacrée à la création de deux œuvres québécoises, la Cantate de la fin du jour, d'Isabelle Panneton, et Décimations, de Jacques Desjardins, deux œuvres « à texte », interprétées par l'Ensemble vocal de l'OSM, l'Ensemble Contemporain de Montréal sous la direction de Véronique Lacroix, avec la participation du soprano Marie-Danielle Parent. Le travail d'Isabelle Panneton mérite beaucoup d'éloges, par sa rigueur et sa sensibilité, autant que par le choix d'un texte de Daniel Guénette avec lequel la compositrice possède des affinités évidentes. Décimations, de Jacques Desjardins, est une œuvre honnête qui ne renverse rien, sur un poème un peu simplet et exempt de lyrisme de Renaud Longchamps. Le concept de cet événement, un peu lâche, donnait malgré tout un visage cohérent à l'ensemble des œuvres au programme, qui ont bénéficié de bonnes interprétations. Espérons quand même que le message ne vienne pas à primer sur la musique, dans les événements très « construits » de l'ECM.

\section{La saison de la SMCQ}

Comme chaque année, la Société de Musique Contemporaine du Québec, dans l'esprit d'ouverture et d'honnêteté qui a toujours été le sien, nous a donné à entendre plusieurs aspects de l'évolution récente de la création musicale. En tant que véhicule privilégié de la culture musicale occidentale « contemporaine", la SMCQ offre à l'oreille curieuse un éventail de tendances qui s'entrechoquent ou se côtoient paisiblement. La présentation de chaque événement, d'ailleurs, donnait clairement la piste à suivre : celle de la confrontation positive entre la création musicale québécoise et le répertoire universel de la musique de notre siècle. Québec-Amériques, Québec-France, 
Québec-New York, Québec-Monde et Québec-Allemagne, les cinq concerts de la saison 1992-1993, mettaient en parallèle des œuvres de Québécois, Serge Garant, Serge Provost, René Lussier, Alain Thibault ou Claude Vivier, avec des œuvres européennes, d'Edgard Varèse, Luc Ferrari, Karlheinz Stockhausen ou Nicolaus A. Huber, par exemple.

\section{Québec-Amériques}

Le soir du 22 octobre 1992, Québec-Amériques mettait en scène le merveilleux accordéoniste torontois Joseph Petric, l'altiste Marc Bélanger et le guitariste électrique René Lussier, avec la participation de l'Ensemble de la SMCQ sous la direction de Walter Boudreau. Circuit II de Serge Garant, arghanum I d'Alcides Lanza, The Viola in My Life II de Morton Feldman, Destination Soleil... de René Lussier (en création ce soir-là), et Déserts d'Edgard Varèse étaient au programme. Entre Garant et Varèse, tous deux interprétés avec intensité et respect, les autres compositeurs se partageaient les trois instruments nommés plus haut. Lanza l'accordéon, Feldman l'alto, et Lussier, bien sûr, la guitare. L'œuvre de Lanza, pour sa part, était intéressante et loin de présenter l'accordéon comme une simple machine à faire du bruit. Il va sans dire que le talent de Petric, qui sait faire chanter l'instrument d'une manière tout à fait surprenante, contribuait beaucoup à rendre la chose musicale. Feldman bénéficia également d'une interprétation fort convenable de Marc Bélanger. La grosse surprise du concert était la prestation de René Lussier, musicien essentiellement improvisateur, qui avait réellement composé, pour l'occasion, une œuvre le mettant en évidence, sans toutefois le mettre en valeur. On ne peut rien reprocher à Lussier, dans ce contexte tout à fait particulier d'un concert presque "classique». On ne peut pas non plus reprocher à la $S M C Q$ de nous mettre en face d'un genre de musique issue d'une fusion entre divers styles, qu'ils soient de souches populaires ou peut-être un peu varésienne... Mais il faut se rendre à l'évidence, l'effet n'était pas des plus réussis. Déserts, par ailleurs, était superbe, et le nettoyage de la bande - exécuté par Alain Thibault -, donnait une allure de jeunesse à cette œuvre qui paraissait avoir prématurément vieilli.

\section{Québec-France}

Louise-André Baril, au piano, Jean-Guy Plante, Robert Leroux et Serge Desgagnés aux percussions, donnaient le 10 décembre 1992 un concert qui 
avait en seconde partie des allures de spectacle. Talea, de Grisey, Le Loriot, extrait du Catalogue d'oiseaux de Messiaen, et en création, Églogue-Le jardin des oliviers de Serge Provost formaient la première partie de cet événement dédié à la "douce mémoire d'Olivier Messiaen», - dont c'était à cette date l'anniversaire de naissance. C'est dans le plus grand sérieux que ce concert avait donc débuté, avec la création d'une œuvre à la facture presque classique et néanmoins moderne, très bien construite et qui s'exprimait par la musique seule. Société II, de Luc Ferrari, apportait une toute autre coloration à l'événement, en insérant dans l'œuvre musicale une dimension métaphorique, manquant malheureusement beaucoup de subtilité. La placide pianiste Louise-Andrée Baril y subissait les assauts musicaux de trois mâles percussionnistes, censés incarner (mais ce n'est pas sûr...) l'attitude macho. Société II Et si le piano était un corps de femme pourrait peut-être intéresser les amateurs de lieux communs sur les rapports amoureux, mais certainement pas les mélomanes.

\section{Québec-New York}

Le flûtiste new yorkais Robert Dick est tout à fait ce que l'on imagine du musicien d'avant-garde américain: dové, opportuniste, plutôt infantile et très imaginatif. Ce troisième concert de la saison de la $S M C Q$, le 21 janvier 1993, lui était entièrement consacré, à lui et à ses "œuvres», à l'exclusion d'une pièce de Marc Hyland, Siegfried-Tableaux, qui était jouée ce soir-là en création. Afin de s'assurer de la présence d'une œuvre québécoise dans le programme de ce concert, la SMCQ avait auparavant lancé un concours d'œuvres pour flûte solo, dont le grand prix serait d'être joué par le flûtiste Robert Dick... Dans le programme, on nous annonçait la possibilité d'entendre James Harley, Pierre-Kresimir Klanac et Marc Hyland, qui se disputaient l'honneur de cette interprétation prestigieuse. Le nom du gagnant ne fut annoncé qu'à la dernière minute, afin de créer le suspense. Ce fut un soulagement $d^{\prime}$ entendre, après cinq pièces de Dick et une interminable improvisation, une œuvre structurée et réfléchie. Dick est certes un grand virtuose, qui soutire à son instrument des sonorités étonnantes et fort belles. Mais le charme est de courte durée, et le manque d'épine dorsale de ses compositions conduit rapidement à l'ennui, sinon à l'irritation. Robert Dick compose comme il improvise, et improvise comme il compose, sans chercher autre chose que le plaisir immédiat et ludique de produire des sons. Son succès et sa notoriété sont assez symptomatiques à cet égard. 


\section{Québec-monde}

Ce concert réunissait, le 25 mars 1993, deux interprètes de qualité ayant une vaste expérience de la création musicale: le percussionniste Robert Leroux et le pianiste Louis-Philippe Pelletier, qui ont offert au public un concert chaleureux et sans prétention. Champs I, de Gilles Tremblay, Tsi-Shin/TaRyong III de Younghi Pagh-Paan, Out d'Alain Thibault et Kontakte de Karlheinz Stockhausen formaient un beau panorama musical qui nous faisait entendre des œuvres de plusieurs pays et de plusieurs allégeances. La plus dissidente était sans aucun doute Out, de Thibault, pour percussion midi et bande. Si Thibault veut nous faire prendre conscience du phénomène de la politique-spectacle, sa musique doit être exécutée à la perfection pour permettre au message de dépasser le niveau de la simple référence anecdotique. Elle le fut ce soir-là, et suscita, sans être une musique engagée, une réflexion sur un phénomène social important. Interprété avec intelligence et virtuosité, Out devient d'un raffinement étonnant, et utilise à des fins de communication la sensation physique créée par des rythmes trépidants, sans tomber dans l'assourdissant et le vulgaire. Thibault n'est peut-être pas un grand compositeur, mais ses œuvres trahissent l'exigence et le métier, tout comme le jeu de Robert Leroux.

\section{Québec-Allemagne}

Stockhausen, cette figure immense de l'avant-garde des années 1950-1970, terminait le précédent concert de la SMCQ, et débutait le dernier de la saison: le compositeur allemand faisait ainsi le pont entre Québecmonde et Québec-Allemagne. L'Ensemble de la SMCQ, sous la direction de Walter Boudreau, a interprété le 6 mai 1993 Kreuzspiel, de Stockhausen, Sechs Bagatellen de Nicolaus A. Huber, Mysterium, de Wim Henderickx ef Liebesgedichte de Claude Vivier. Si la première pièce du concert était consistante, les six bagatelles de Huber nous ont semblé légères et assez inintéressantes, bien que très allemandes dans l'esprit. Mysterium, Prix FlandreQuébec 1991, est quant à elle une très belle œuvre pleine de raffinement, qu'il faudra réentendre. Il est à déplorer que l'œuvre de Vivier ait eu à subir une mauvaise interprétation, surtout de la part des chanteurs qui, semble-t-il, n'étaient pas tout à fait présents, ce soir-là, à ce qu'ils faisaient. 


\section{PRÉSENCES}

Présences, semaine de musique contemporaine québécoise, offrait du 16 au 23 mai 1993 une fin de saison très riche au public montréalais, en soulignant avec effervescence le vingtième anniversaire du Centre de musique canadienne au Québec. Ici, plus question de courants ou de sélection stylistique; la volonté des organisateurs était de présenter l'éventail des manières d'aborder la musique au Québec, depuis l'avènement de la modernité. Le passé y côtoyait donc le présent, la musique d'improvisation s'y frottait au structuralisme, et la création électroacoustique y avait également une place importante. Rencontres, conférences et concerts étaient au programme, avec des invités de choix et la participation de tous les ensembles et sociétés de concert les plus actifs sur la scène montréalaise, dans le domaine de la création musicale. Le Nouvel Ensemble Moderne, l'Association pour la création et la recherche électroacoustique du Québec, les Productions Supermémé, I'Ensemble de la SMCQ, "Codes d'Accès» et le Quatuor Morency donnaient chacun un concert complet, créant presque une saison musicale en microcosme. Comme dans toutes les saisons, d'ailleurs, certains événements avaient plus à offrir que d'autres.

Un des concerts les plus intéressants de cette semaine intense - et qui ouvrait d'ailleurs Présences -, mettait de l'avant une idée originale et pleine de fraîcheur. Le Concours CMC-Vincent-D'Indy présentait en récital ses lauréats, dans des œuvres composées pour l'événement par des compositeurs d'ici, anciens élèves de cette réputée école de musique montréalaise. Cinq créations étaient au programme, interprétées par cinq musiciens et musiciennes de moins de vingt ans. La rencontre entre ces jeunes interprètes et la musique des compositeurs québécois des années 1990 avait quelque chose de piquant, de nouveau, de vivifiant. Michel Gonneville, Denis Gougeon, Isabelle Panneton, Nicole Rodrigue et Karl-Hugo Vande Kerckhove s'étaient tous les cinq limités à un langage simple, accessible, à une écriture exempte de trop grandes difficultés d'interprétation. On pardonne alors facilement le côté peut-être un peu enfantin de certaines de ces compositions. Par ailleurs, l'enthousiasme des jeunes musiciens devant ces œuvres «vivantes" était tel qu'il justifiait cette démarche simplificatrice. Promenade avec l'anarchiste, de Gonneville, pour piano et bande, Le Jeu des citations, de Gougeon, pour voix et piano, Les Petites Reprises, pour marimba, de Panneton, Le Moqueur Polyglotte de Rodrigue et Sortilège de Vande Kerckhove formaient ce corpus 
d'œuvres qui, souhaitons-le, servira souvent aux jeunes musiciens québécois de porte d'entrée dans le monde musical contemporain.

Les productions Supermémé, quand à elles, présentaient un concert sous le signe de l'improvisation, qui incarnait à lui seul tout ce pan de la musique québécoise qualifiée d'actuelle. II y a peu à dire sur une musique qui n'a ni logique ni discours, et dont le but est certainement beaucoup plus de nous déstabiliser que de nous charmer. La présence d'un thème, s'il inspire les musiciens, qui ici, notons-le, ne sont pas des « interprètes», n'ajoute aucune cohérence apparente au déroulement des improvisations. Le résultat est parfois intriguant, souvent irritant, toujours évanescent, puisqu'il ne s'agit que d'occuper provisoirement l'espace sonore, sans intention de pérennité. Peut-être, plus que toute autre, cette « musique » exprime-t-elle le profond malaise culturel que nous vivons.

L'Ensemble de la SMCQ donnait, dans le cadre de cette semaine de Présences, un concert qui se plaçait au contraire dans une continuité tout à fait palpable, celle de la modernité musicale québécoise, descendante directe du courant européen, lui-même inscrit dans une tradition séculaire. Les six compositeurs au programme montraient pourtant chacun un aspect différent de cette modernité, formant ainsi un portrait de groupe tout à fait réussi du Québec musical des deux dernières décennies. Serge Garant, Walter Boudreau, Gilles Tremblay, Micheline Coulombe Saint-Marcoux, John Rea ef Claude Vivier ont à cette occasion bénéficié d'interprétations de qualité.

Le concert de la société "Codes d'Accès», qui représente en quelque sorte le mi-chemin entre la musique contemporaine et la musique actuelle, a pour sa part offert une image peu flatteuse de ce que les jeunes compositeurs québécois peuvent produire pour un événement comme cette semaine de musique contemporaine québécoise. Encore une fois, ils ont déçu, par leur manque de respect pour l'auditeur et le manque de ressources dont il font preuve devant une feuille vierge de papier à musique...

Malgré cette déception, l'aspect inquiétant de l'année musicale 1992-1993 n'apparaissait aucunement à travers les différents événements de cette semaine d'effervescence musicale, et laissait plutôt croire à une renaissance de la confiance des créateurs en leur potentiel d'espérance. Des semaines comme celle-ci ont le mérite de redonner aux auditeurs et aux musiciens la sensation que tout est encore possible... 


\section{Espoir, encore}

Une pointe d'optimisme nous laisse donc espérer que l'avant-garde n'est que temporairement occultée, puisqu'elle laisse surgir au travers de l'épaisse couche de poussière qui la recouvre certaines œuvres dignes d'une admiration inconditionnelle. Entre Avec, Wampum symphonique, de Gilles Tremblay, la Messe sur le monde de Clermont Pépin, la rétrospective Kagel et l'hommage à Xenakis du Nouvel Ensemble Moderne, une grande œuvre est née de la plume d'un compositeur au seuil de la quarantaine: L'adorable verrotière, de Serge Provost, sur un texte de Claude Gauvreau. N'oublions pas toutefois de mentionner un événement considérable - auquel, malheureusement, nous n'avons pu assister - et qui n'avait rien d'extra-musical, puisqu'il s'agissait de la création, par le magnifique Quatuor Arditti, d'une œuvre du compositeur montréalais John Rea. Le quatuor Objets perdus de Rea a obtenu, à juste titre, en 1992, le Prix Jules-Léger pour la nouvelle musique de chambre, récompense prestigieuse, qui était pour la seconde fois accordée à ce compositeur, représentant essentiel de la sensibilité de son époque. Par ailleurs, l'absence de Forum - événement biennal organisé par le NEM - en 1992 a contribué à accentuer cette sensation de retour en arrière. Un automne sur deux, les années impaires, une partie importante des ressources dépensées à Montréal dans le domaine de la création le sont pour ce forum réunissant de jeunes compositeurs de différents pays. Les années paires, toujours à l'automne, l'ensemble rend au contraire hommage à un grand créateur ayant marqué l'avant-garde musicale, mouvement qui a connu son heure de gloire entre 1950 ef 1970... A Montréal, c'est donc artificiellement que l'on renforce l'effet de balancier « regard vers l'avenir /regard vers le passé „... Mais ne confondons pas le fait de rendre un hommage mérité à ceux qui ont osé, et les gestes créatifs volontairement rétrogrades qui ont marqué cette dernière saison.

On est en droit de se demander si, néanmoins, ce mouvement ne vaut pas aussi bien que «l'idolâtrie des valeurs juvéniles" qu'évoque avec hantise le philosophe Alain Finkielkraut (1987). Cet envahissement progressif de la culture par l'insignifiance, le «cucul», le facile, dont nous sommes depuis quelques temps les victimes impuissantes (et dont le flûtiste américain Robert Dick fût cette saison, en musique, le digne représentant), menace sérieusement les jeunes créateurs québécois, perméables à cette influence pernicieuse et 
ô combien puissante. Entre l'infantilisme ef la réaction, le compositeur "adulte " commence malheureusement à faire figure de marginal...

FINKIELKRAUT, A., 1987: La défaite de la pensée, Paris, Gallimard.

FISETTE, J., 1992: «Claude Gauvreau : musicien, dramaturge, poète», Circuit, Vol. III, no 2, pp. 7-19.

PROVOST, S., 1992: « Le mot, le son, le sens», Circuit, Vol. III, no 2, pp. 21 -43.

THION, M., 1993: «Les musiques d'aujourd'hui en questions», le Monde de la musique, no 163, pp. 60-66. 


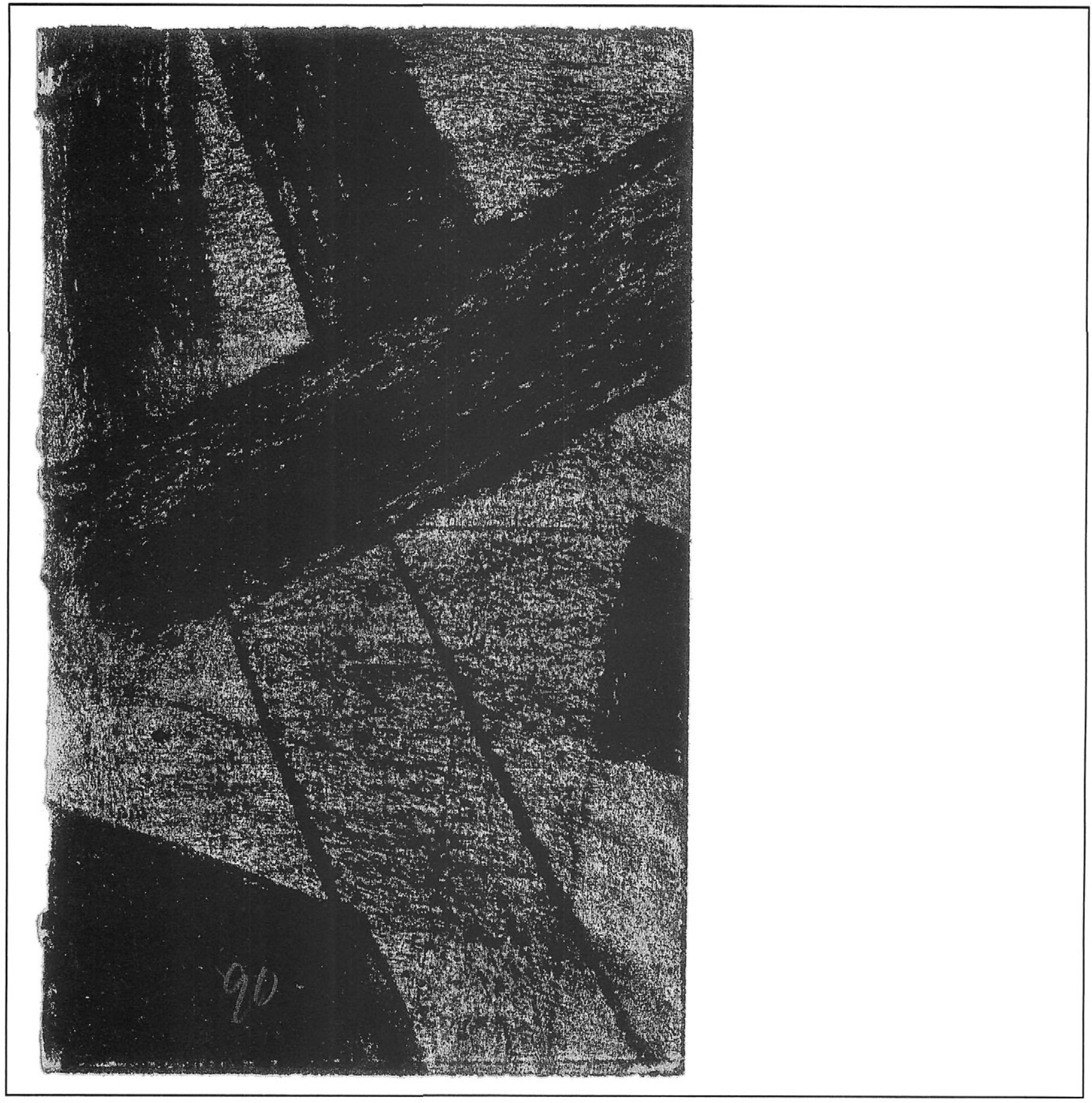

